\title{
Chaos-Based NOMA for Secure Wireless Communications over Rayleigh Fading Channels
}

\author{
Israa Almusawi ${ }^{1}$, Walid Al-Hussaibi ${ }^{2}$, Yaseen H. Tahir ${ }^{3}$ \\ \{israa.almusawii@gmail.com ${ }^{1}$, alhussaibi@stu.edu.iq ${ }^{2}$, yaseen_tcb@stu.edu.iq ${ }^{3}$ \} \\ $\left\{\mathrm{FGS}^{1}, \mathrm{BTI}^{2}, \mathrm{QTI}^{3}\right\}$, Southern Technical University, Basrah, Iraq
}

\begin{abstract}
Non-orthogonal multiple access (NOMA) is considered as one of the key technologies to achieve the main targets of fifth generation $(5 \mathrm{G})$ wireless networks and beyond such as high user connectivity and ultra-reliability. However, as the number of simultaneously served users increased, the security of users' data becomes a real concern due to high possibility of unauthorized interception. To mitigate the critical security issue, diverse chaos-based communication systems have been introduced in the literature to provide cost-effective solutions for physical layer security. In this paper, an efficient chaosbased NOMA (CB-NOMA) scheme is proposed for secure wireless transmission. The integrated CB-NOMA design is implemented based on chaotic code-domain with equal power allocation for connected users over Rayleigh fading environment. Different chaoticcode formation scenarios with diverse security levels and implementation complexity are considered for chaos shift keying signalling. Intensive simulation results validate the effectiveness of proposed CB-NOMA compared with the considered reference systems. It shows direct relation between the significant improvement in error performance and utilized lengths of chaotic codes apart from employed code generation source. Besides, beneficial tradeoffs are demonstrated between the achieved error rate, user connectivity, security level, and system complexity. Thus, the presented CB-NOMA can be seen as a promising candidate for future secure wireless applications.
\end{abstract}

Keywords: Chaotic communications; code-domain NOMA; wireless systems; physical layer security; Rayleigh fading channel.

\section{Introduction}

The huge digital revolution in information technology worldwide has led to massive increase in smart mobile phones/tablets/laptops, unmanned aerial vehicles, wireless sensors, and Internet of things (IoT) devices. Consequently, the new communication networks are anticipated to fulfil the growing burdens for wireless services with critical requirements of high user connectivity, ultra-reliability, low-latency, robust data security, low complexity, and affordable implementation cost [1]-[5]. For conventional orthogonal multiple access (OMA) schemes like frequency division multiple access (FDMA), time division multiple access (TDMA), code division multiple access (CDMA) and orthogonal frequency division multiple access (OFDMA), the number of supported users is limited by the available number of orthogonal resources [2], [6]. To overcome this critical problem, non-orthogonal multiple access (NOMA) concept based on the code-domain and/or power-domain has been considered as one of the essential techniques to achieve the core objectives of fifth generation $(5 \mathrm{G})$ wireless systems and beyond [1], [3]. In such model, the allowed users can utilize the available non-orthogonal 
resources (i.e. non-orthogonal codes and/or distinct power level), but at cost of unavoidable cochannel interference and/or error propagation in signal detection [2], [3], [5], [6].

In power-domain NOMA, the connected users are assigned different power levels based on their channel conditions. For instance, downlink users with poor channel gains are assigned more transmitted power than the users with strong channel gains. Successive interference cancellation (SIC) technique can be used then at the receiver to separate the transmitted users' signals [1]. But in the uplink channel, the weak users may use low transmission power to prolong the lifetime of their batteries and satisfy the power difference condition with strong users for effective SIC procedure [3]. In code-domain NOMA, the power difference between served users is not mandatory since the users' signals are separated through non-orthogonal spreading sequences as in low-density spreading CDMA (LDS-CDMA), sparse code multiple access (SCMA), and multiuser shared access (MUSA) schemes [2]. It should be noted that NOMA strategy can also be achieved using two sets of orthogonal signal waveforms as in the pioneer work of Sari et al. in [6] and other recent results in [4] and [7].

On the other hand, the increased number of simultaneously connected users in NOMA schemes may lead to serious security problems in users' transmitted data. To mitigate this critical issue, varied energy-efficient chaos-based secure communication (CBSC) systems have been suggested by the research and industrial communities to provide cost-effective physical layer security for emerging wireless application [8]-[11]. These systems are designed by exploiting the admirable features of chaotic signals such as the high sensitivity to initial conditions (ICs), broadband spectrum, unpredictability, simple generation using inexpensive electronic circuits, and high immunity to jamming and interference [12]-[14]. The information messages in CBSCs are typically concealed into the employed chaotic signal and transmitted to the destination node over wireless channel environment. For coherent signal detection, chaos synchronization between transmit and receive ends is essential to restore the transmitted messages [13]. But, this prerequisite is not needed in non-coherent receivers where data recovery can be achieved by observing the received signal features [15], [16].

By exploiting the fabulous broadband property of chaotic signals, as in spread spectrum communications, numerous CBSC schemes have been proposed for the multiple access channel over the past years [8]-[10], [16]-[19]. A chaos based CDMA was proposed in [19] by employing the generated chaotic signals from same Chua's circuit but with different ICs to spread the users' data. It was found that two-fold of conventional CDMA capacity can be achieved at lower implementation cost. In [9], chaos and NOMA are combined in a grant-free sparse chaos code multiple access (GF-SCCMA) technique. In this system, the generated chaotic signals with different ICs are used for the construction of classical SCMA codebook. For these systems of extended capacity, each user has a unique IC which plays the crucial role of encryption key for robust physical layer security where intended receiver with the right IC can decode the received data. Chaos shift keying (CSK) and differential CSK (DCSK) modulation techniques are used also for the multiple access channels in [17] and [18], respectively. However, the performance of aforementioned multiuser systems is demonstrated over the simple scenario of Gaussian channel which affects the feasibility of presented designs. In [10], chaos coding has been used in the multiple-input multiple-output (MIMO) for uplink (UL) CBSC with power-domain NOMA in some of the utilized subcarriers for higher security, capacity, and connectivity, but at cost of decoding complexity. Chaos-based power-domain MIMO-NOMA is also investigated in [8] for the downlink (DL) fading channel with security enhancement. However, the employment of the well-known MIMO and orthogonal frequency division multiplexing (OFDM) technologies in [8] and [10] may have the effect to exaggerate the performance of chaos-based security. 
In this paper, an uplink chaos-based NOMA (CB-NOMA) is proposed for coherent CBSC systems over wireless fading channel. The supported users employ CSK signalling for codedomain NOMA with equal power allocation to provide the important physical layer security. Diverse generation approaches for utilized chaotic signals with different codes lengths are investigated for the designed CB-NOMA scheme considering joint maximum likelihood (ML) receiver for multiuser detection (MUD). The main contributions of this research are highlighted as follows:

1) A system design of an uplink code-domain CB-NOMA is presented including complete mathematical signal model over realistic wireless Rayleigh fading channel rather than the simple Gaussian environment in [17] and [18].

2) For efficient performance evaluation, different scenarios are considered for the generation of chaotic-codes with various security levels. These codes are employed at the adopted CSK and modified CSK (MCSK) modulators/demodulators.

3) The effectiveness of designed CB-NOMA is validated through extensive simulations of bit-error-rate (BER) compared with the reference systems by isolating the well-known gains of OMA schemes such as multiuser MIMO and OFDM in [8] and [10]. The achieved outcomes are very interesting and demonstrated useful tradeoffs between the achieved BER, desired security level, user connectivity, and system complexity.

The rest of this paper is organized as follows. Brief technical background on the generation of chaotic signals is given in Section 2. System design of CB-NOMA and the considered mathematical signal model are presented in Section 3. The conducted simulation results are depicted in Section 4. Finally, Section 5 concludes the paper and highlights the future work.

\section{Background on the Generation of Chaotic Signals}

In the field of CBSC, chaotic signals are commonly used for different modulation techniques such as chaos shift keying (CSK), chaotic masking (CM), chaos on-off keying (COOK), and chaos parameter modulation (CPM) [15]-[18]. The adopted chaotic signals can be generated using very simple (inexpensive) electronic circuits that demonstrate different nonlinear dynamical systems such as Lorenz chaotic system (LCS) [13], Chua chaotic system (CCS) [15], and Henon chaotic system (HCS) [20]. Besides, the mathematical models of these dynamical systems can be solved using many computer programs such as MATLAB [15].

\subsection{Lorenz Chaotic System (LCS)}

This three-dimensional nonlinear system (chaotic attractor) is described mathematically by the following ordinary differential equations [13]

$$
\begin{aligned}
& \dot{x}=\sigma(y-x) \\
& \dot{y}=r x-y-x z \\
& \dot{z}=x y-b z
\end{aligned}
$$

where $x, y$, and $z$ represent the states of LCS, $\sigma=10, r=28$, and $b=8 / 3$. Using computer simulation, the dynamical system (1) can be solved to find chaotic signals $x, y$, and $z$. Figure 1 demonstrates the time waveform segments of generated states $(x, y$, and $z)$ and sensitivity to ICs using two different sets of considered values. 

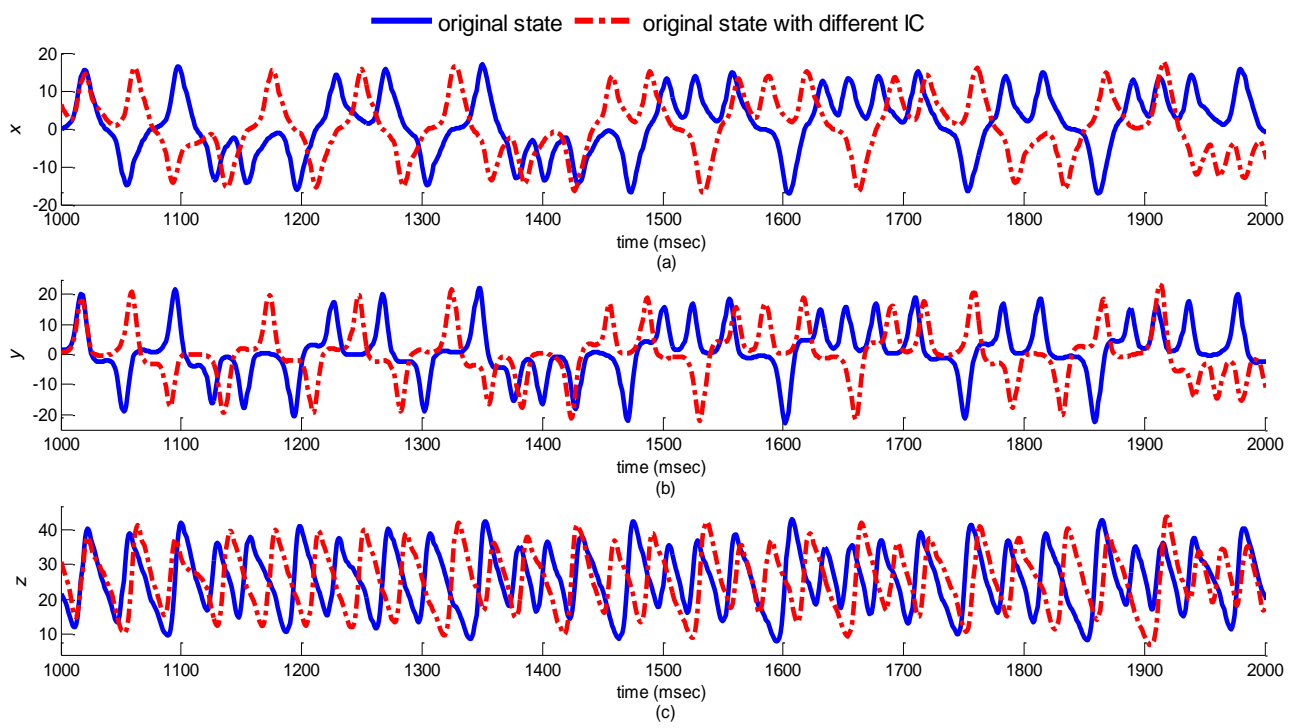

Fig. 1.Time waveform segments of the generated states by LCS with two different sets of ICs. (a) $x$ state, (b) $y$ state, and (c) $z$ state.

\subsection{Chua Chaotic System (CCS)}

This three-dimensional nonlinear system can be used to generate one of the typical chaotic attractors denoted as the double scroll attractor and described by the following ordinary differential equations [15].

$$
\begin{aligned}
& \dot{x}=\left[G(y-x) / C_{1}\right]-\left[g(x) / C_{1}\right] \\
& \dot{y}=\left[G(x-y) / C_{2}\right]+\left[z / C_{2}\right] \\
& \dot{z}=-y / L
\end{aligned}
$$

where $g(x)=m_{0} x+\left(m_{1}-m_{0}\right)\left(\left|x+B_{P}\right|-\left|x-B_{P}\right|\right) / 2$, and the utilized parameters for chaotic signal behaviour are $C_{1}=1 / 9, C_{2}=1, L=1 / 7, G=0.7, m_{0}=-0.5, m_{1}=-0.8$, and $B_{P}=1$. By simulation, (2) can be solved to find the chaotic signals $x, y$, and $z$ of CCS. In Figure 2 (a), without loss of generality, a segment of the chaotic time waveform $x$ is shown for two different arrangements of ICs.

\subsection{Henon Chaotic System (HCS)}

The attractor of this two-dimensional nonlinear system can be described using the following set of difference equations of discreet-time instance $n$ [20].

$$
\begin{aligned}
& x(n+1)=y(n)+1-a x^{2}(n) \\
& y(n+1)=b x(n)
\end{aligned}
$$

where the considered parameters for chaotic system are selected as $a=1.4$ and $b=0.3$. Through numerical simulation, (3) can be solved to find the chaotic states $x$ and $y$ of HCS. Figure 2 (b) shows a segment of the time sequence of generated state $x$ with two possible sets of ICs. 

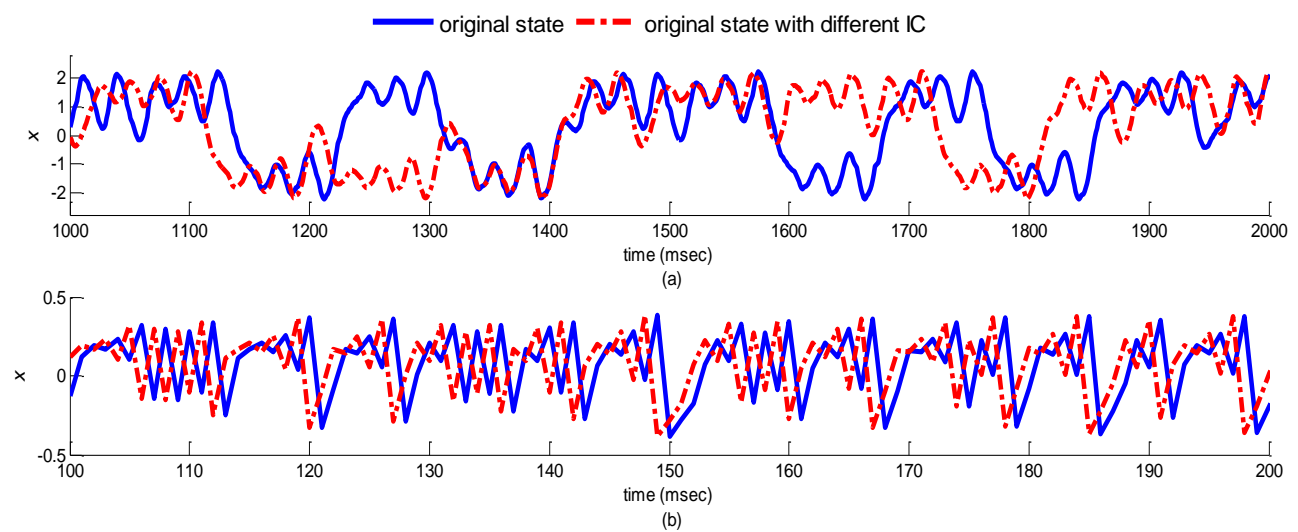

Fig. 2.Time waveform segments of the generated $x$ states with two different sets of ICs using (a) CCS and (b) HCS.

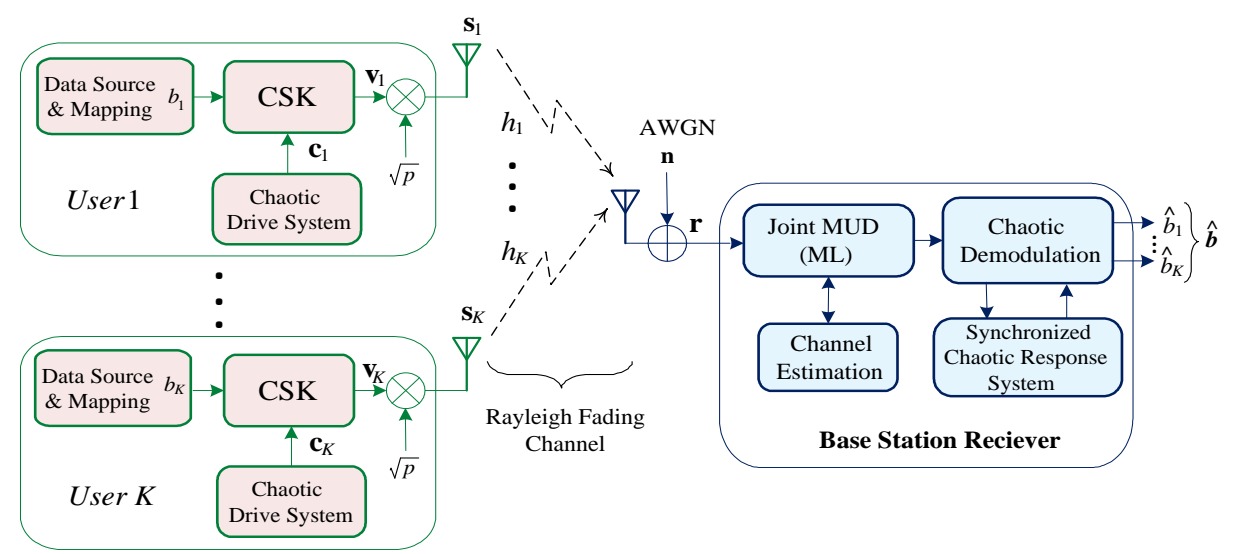

Fig. 3. System design of a generalized code-domain CB-NOMA with CSK signalling over wireless Rayleigh fading channel.

\section{System Design of CB-NOMA}

Consider a generalized code-domain CB-NOMA of $K$ single-antenna users communicating simultaneously with a common single-antenna base station (BS) over wireless Rayleigh fading channel as shown in Figure 3. Synchronized chaotic systems, in drive-response fashion [13], are adopted at the transmitters and BS receiver for CSK modulation and demodulation, respectively. For CSK, different chaotic systems such as LCS, CCS, and HCS are used for the generation of spreading codes. At the BS, the optimal joint ML receiver is employed for MUD assuming perfect channel state information (CSI) through channel estimation unit. Note that the adopted design of CB-NOMA in its basic configuration is very necessary to separate the wellknown gains of other integrated OMA techniques like multiuser MIMO and OFDM [8], [10], and then provides a realistic performance assessment. 


\subsection{Signal Model}

At the transmit side, the digital information data of user $k$ is mapped into antipodal (binary) bits $b_{k} \in\{+1,-1\}_{k=1}^{K}$ and modulated using CSK with a specific real chaotic code $\mathbf{c}_{k} \in \mathcal{R}^{1 \times \beta}$ of length $\beta$ chips (from any state of chaotic system like LCS, CCS, or HCS) as

$$
\mathbf{v}_{k}=b_{k} \mathbf{c}_{k}=b_{k}\left[\begin{array}{lll}
c_{k, 1} & \ldots & c_{k, \beta}
\end{array}\right]=\left[\begin{array}{lll}
v_{k, 1} & \ldots & v_{k, \beta}
\end{array}\right]
$$

where $c_{k, i}$ is the $i^{t h}$ chip of assigned chaotic code $\mathbf{c}_{k}$ for user $k, v_{k, i}$ is the $i^{\text {th }}$ chip of modulated signal vector $\mathbf{v}_{k} \in \mathcal{R}^{1 \times \beta}$ of user $k$ with normalized power, and the bit duration $T_{b}$ is assumed to be $\beta$ times chip duration $T_{c}$ (i.e. $T_{b}=\beta T_{c}$ ).

Based on the formation approach of utilized chaotic code $\mathbf{c}_{k} ; k=1, \ldots, K$, two convenient strategies for CSK modulation will be investigated. The first CSK modulation technique considers the chaotic sequence of continuous-values as generated from chaotic system where the $i^{\text {th }}$ chip of $\mathbf{v}_{k}$ is represented as

$$
v_{k, i}= \begin{cases}+c_{k, i} ; & \text { for bit" }{ }^{\prime \prime} \\ -c_{k, i} ; & \text { for bit" }-1 " .\end{cases}
$$

The second technique, denoted in this work as a modified CSK (MCSK), implements hard quantization (i.e. $\mp 1$ ) on the original chaotic sequence to realize discrete-values as in CDMA codes. In this case, the $i^{\text {th }}$ chip of $\mathbf{c}_{k}$ is given as

$$
c_{k, i}= \begin{cases}+1 ; & \text { if } \quad c_{k, i} \geq 0 \\ -1 ; & \text { if } \quad c_{k, i}<0\end{cases}
$$

Equal transmit power strategy $(p)$ is adopted since the information signals of connected users are multiplexed in the code-domain. Consequently, the transmitted real signal vector $\mathbf{s}_{k} \in$ $\mathcal{R}^{1 \times \beta}$ of user $k$ is givenby

$$
\mathbf{s}_{k}=\sqrt{p} \mathbf{v}_{k}=\left[\begin{array}{lll}
s_{k, 1} & \ldots & s_{k, \beta}
\end{array}\right] .
$$

The received signal model of CB-NOMA can be given then by

$$
\mathbf{r}=\sum_{k=1}^{K} h_{k} \mathbf{s}_{k}+\mathbf{n}=\mathbf{h} \mathbf{S}+\mathbf{n}
$$

where $\mathbf{r}=\left[r_{1} \ldots r_{\beta}\right] \in \mathcal{C}^{1 \times \beta}$ is the received complex superimposed signal vector, $h_{k}$ is a complex-valued Rayleigh fading channel of zero-mean and unit-variance which assumed to be fixed over the entire bit duration $T_{b}$, and $\mathbf{n}=\left[n_{1} \ldots n_{\beta}\right] \in \mathcal{C}^{1 \times \beta}$ is i.i.d. complex AWGN vector of zero-mean and $\sigma_{n}^{2}$-variance elements, $\mathbf{h}=\left[h_{1} \ldots h_{K}\right] \in \mathcal{C}^{1 \times K}$ is the complex fading channel vector of $K$ user system, $\mathbf{S}=\left[\mathbf{s}_{1} \ldots \mathbf{s}_{K}\right]^{\mathrm{T}} \in \mathcal{R}^{K \times \beta}$ is the overall transmitted signal matrix, and $[.]^{\mathrm{T}}$ stands for the transpose operation.

\subsection{Receiver}

At the receive side, MUD is carried out jointly using the optimal ML which minimize the error probability based on the minimum distance criterion between received signal and all possible signal vector combinations over the associated channels. For the considered CSK 
signalling schemes, there is a set of $\Omega=2^{K}$ probable arrangements for transmitted signal matrix at the channel input as $\mathbb{S}=\left\{\mathbf{S}^{(1)}, \ldots, \mathbf{S}^{(l)}, \ldots, \mathbf{S}^{(\Omega)}\right\}$, where $\mathbf{S}^{(l)}=\left[\mathbf{s}_{1}^{(l)} \ldots \mathbf{s}_{K}^{(l)}\right]^{\mathrm{T}}$, and $\mathbf{s}_{k}^{(l)}$ is the $l^{\text {th }}$ likely transmitted signal vector from user $k$. Thus, the transmitted signal matrix can be estimated at the receiver as

$$
\widehat{\mathbf{S}}=\left[\begin{array}{lll}
\hat{\mathbf{s}}_{1} & \ldots & \hat{\mathbf{s}}_{K}
\end{array}\right]^{\mathrm{T}}=\arg \min _{\mathbf{S}^{(l)} \in \mathbb{S}}\left\|\mathbf{r}-\mathbf{h} \mathbf{S}^{(l)}\right\|^{2}
$$

where $\|$.$\| is the Euclidean norm. The output of estimated users' data can be found then by$ remapping $\left[\begin{array}{lll}\hat{\mathbf{s}}_{1} & \ldots & \widehat{\mathbf{s}}_{K}\end{array}\right]^{\mathrm{T}}$ into $\hat{\mathbf{b}}=\left[\begin{array}{lll}\hat{b}_{1} & \ldots & \hat{b}_{K}\end{array}\right]^{\mathrm{T}} \in \mathcal{R}^{K \times 1}$.

\subsection{Code Formation Scenarios}

For CB-NOMA with desired physical layer security level and reasonable complexity, numerous chaotic code formation scenarios (i.e. $\mathbf{c}_{k} ; k=1, \ldots, K$ of $\beta$ spreading chips) can be used for the implemented modulation techniques (CSK and MCSK). In particular, we consider the following four scenarios and show their practical advantages and limitations.

Scenario 1 (S1). All supported users employ a similar chaotic drive system with the same ICs. The users' codes are formed from the same state (such as the $x$-dimension of LCS), but from different samples of length $\beta$ (i.e. different intervals). In this case, low hardware complexity is achieved at the BS receiver since one chaotic circuit is needed. But, the information security depends mainly on the length of spreading code. This makes the system rather vulnerable to possible attack from intruders/unauthorized clients due to one-level (1L) security. The maximum number of allowed users $(K)$ depends on the target average BER performance.

Scenario 2 (S2). Connected users utilize similar chaotic drive system with the same ICs, but the users' codes are formed from different states (such as the $x, y$, and $z$ dimensions in LCS). As in $\mathrm{S} 1$, the hardware complexity at the receive side is low owing to the need of one chaotic circuit. But, two-level (2L) security can be established based on the code length and selected chaotic state. Note that the maximum number of supported users $(K)$ is limited by the number chaotic system dimensions. For instance, LCS can support up to $K=3$ users compared with 2 users for HCS.

Scenario 3 (S3). The allowed users employ the same state from a similar chaotic drive system, but the users' codes are formed based on different ICs. Note that the chaotic systems are extremely sensitive to ICs (for any state or all states) which makes the task of signal prediction ultra-high complicated. For example, LCS has sensitivity to ICs of about $10^{-17}, 10^{-15}$, and $10^{-16}$ for $x, y$, and $z$ states, respectively. This has direct impact to enlarge the key-space (security) for each user significantly as a combination process over system chaotic states. In this scenario, the hardware complexity at the BS receiver is low also due to one chaotic circuit requirement. Moreover, robust two-level (2L) security can be accomplished based on the code length and utilized ICs. The maximum number of connected users $(K)$ depends on the desired average BER performance.

Scenario 4 (S4). The served users utilize different chaotic drive systems and the users' codes are configured from any state (such as $x, y$, and $z$ in CCS). The hardware complexity at the receiver is relatively high owing to the need of $K$ chaotic circuit. This increased complexity will provide four-level (4L) security based on the utilized code length, chaotic system, chaotic state, 
and ICs. In this scenario, however, the maximum number of allowed users $(K)$ is limited by the number of different chaotic systems implemented at the BS.

In Table 1, a comparison of the main characteristics of aforementioned scenarios (S1-S4) in CB-NOMA system is given based on the hardware complexity, security level of the physical layer, and maximum number of supported users, considering CSK and MCSK. Note that the complexity and security level of considered scenarios in MCSK is lower than that of CSK due to simple hard quantization circuit.

Table 1. Comparison of the main characteristics of considered scenarios (S1-S4) in CB-NOMA.

\begin{tabular}{c|c|c|c|c}
\hline Modulation & Scenario & Complexity & Security Level & Supported Users $(K)$ \\
\hline \multirow{4}{*}{ CSK } & S1 & Low & $1 \mathrm{~L}$ & Depends on the target BER \\
\cline { 2 - 5 } & S2 & Low & L & $K \leq$ No. of chaotic states \\
\cline { 2 - 5 } & S3 & Low & 2L & Depends on the target BER \\
\cline { 2 - 5 } & S4 & Relatively high & 4L & $K \leq$ No. of chaotic systems \\
\hline \multirow{4}{*}{ MCSK } & S1 & Low & $<1 \mathrm{~L}$ & Depends on the target BER \\
\cline { 2 - 5 } & S2 & Low & $<2 \mathrm{~L}$ & $K \leq$ No. of chaotic states \\
\cline { 2 - 5 } & S3 & Low & $<2 \mathrm{~L}$ & Depends on the target BER \\
\cline { 2 - 5 } & S4 & Moderate & $<4 \mathrm{~L}$ & $K \leq$ No. of chaotic systems \\
\hline
\end{tabular}

\section{Simulation Results}

In this section, numerical simulation experiments are conducted using MATLAB environment to show the error performance and system tradeoffs of designed CB-NOMA using CSK and MCSK for the adopted code formation scenarios (S1-S4). The power constrains for each user is considered as $p=1$, and the achieved BER results are averaged over $10^{6}$ channel realizations. The benchmark results of single-user and multiuser binary phase-shift keying (BPSK) systems and CDMA that employs Walsh-Hadamard sequence (of length equal to 8) are used for reference comparisons. For notational convenience, the outcomes of CSK scheme with code formation scenario S1 is represented as CSK-S1, and so on for other configurations.

Figure 4 shows the average BER as a function of signal-to-noise ratio (SNR) for 2 users system with two cases of spreading code length as $\beta=50$ and $\beta=100$. It can be seen that the BER of all considered system configurations outperform the reference schemes over the entire range of SNR due to code-domain diversity, and the error performance is improved considerably as the code length $(\beta)$ increased. Besides, CSK-S2 scheme achieves superior performance with SNR gain of about $3 \mathrm{~dB}$ compared with CSK-S4 and MCSK-S4 schemes for both $\beta$ values at target BER of $10^{-4}$. These findings are also achieved by CB-NOMA system of 3 users in Figure 5 which demonstrate valuable tradeoffs between target BER performance, complexity, and desired level of physical layer security. For instance, S4 has the lowest BER performance compared with the other scenarios due to utilized nonorthogonal codes from different chaotic systems, and hence user interference, but this BER difference might be considered insignificant for ultra-high security requirements. Summary of the approximate SNR gains of investigated scenarios in Figures $\mathbf{4}$ and $\mathbf{5}$ are presented in Table 2 compared with the reference results of Walsh-Hadamard scheme. 


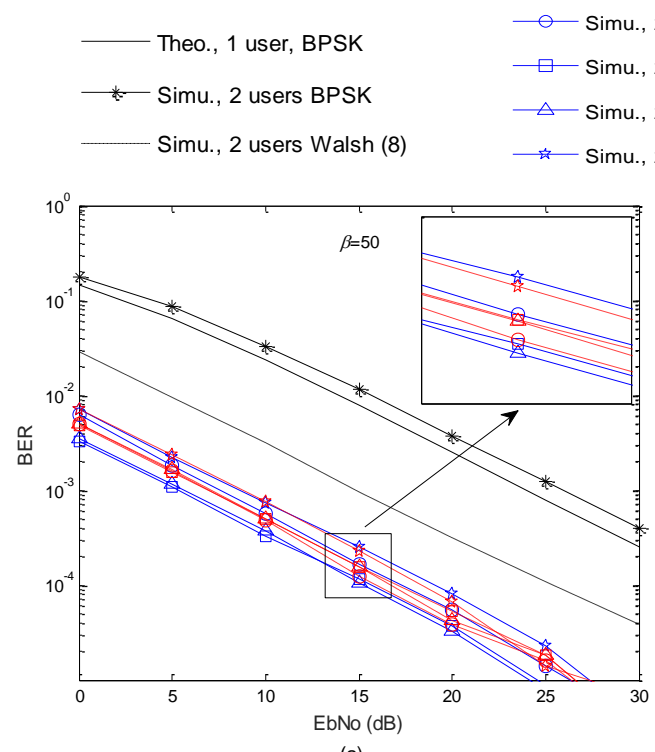

(a)

Fig. 4. Average BER performance of 2 users CB-NOMA over Rayleigh fading channel compared with the reference systems: (a) $\beta=50$, and (b) $\beta=100$.

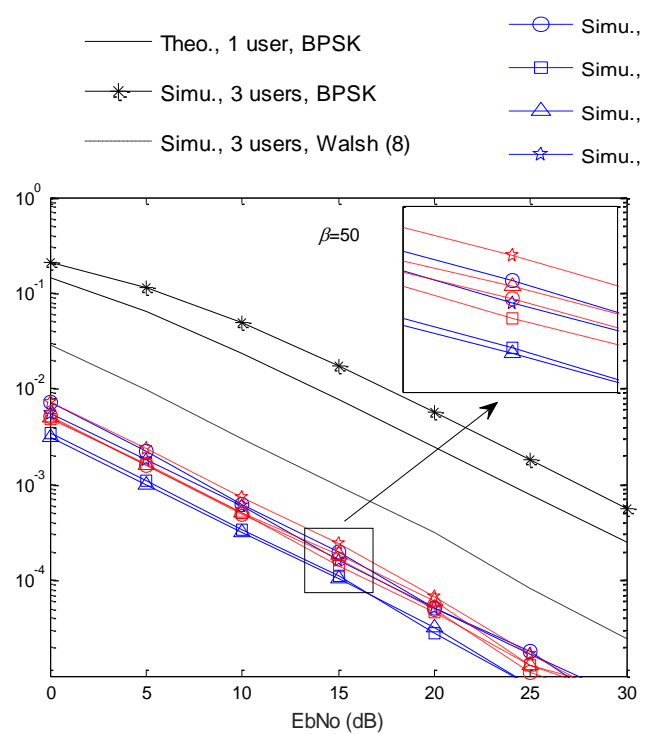

(a)

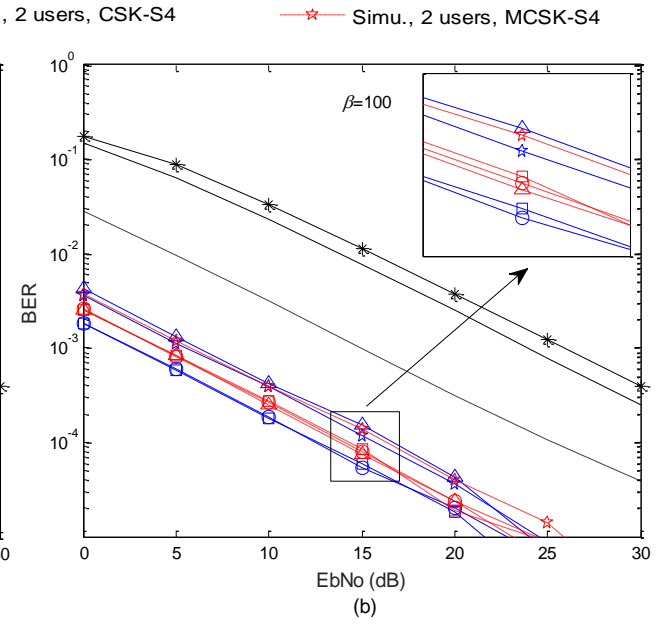

(b)

$\because$ Simu., 2 users, MCSK-S2

Simu., 2 users, MCSK-S3

Simu., 2 users, MCSK-S4

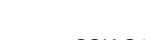




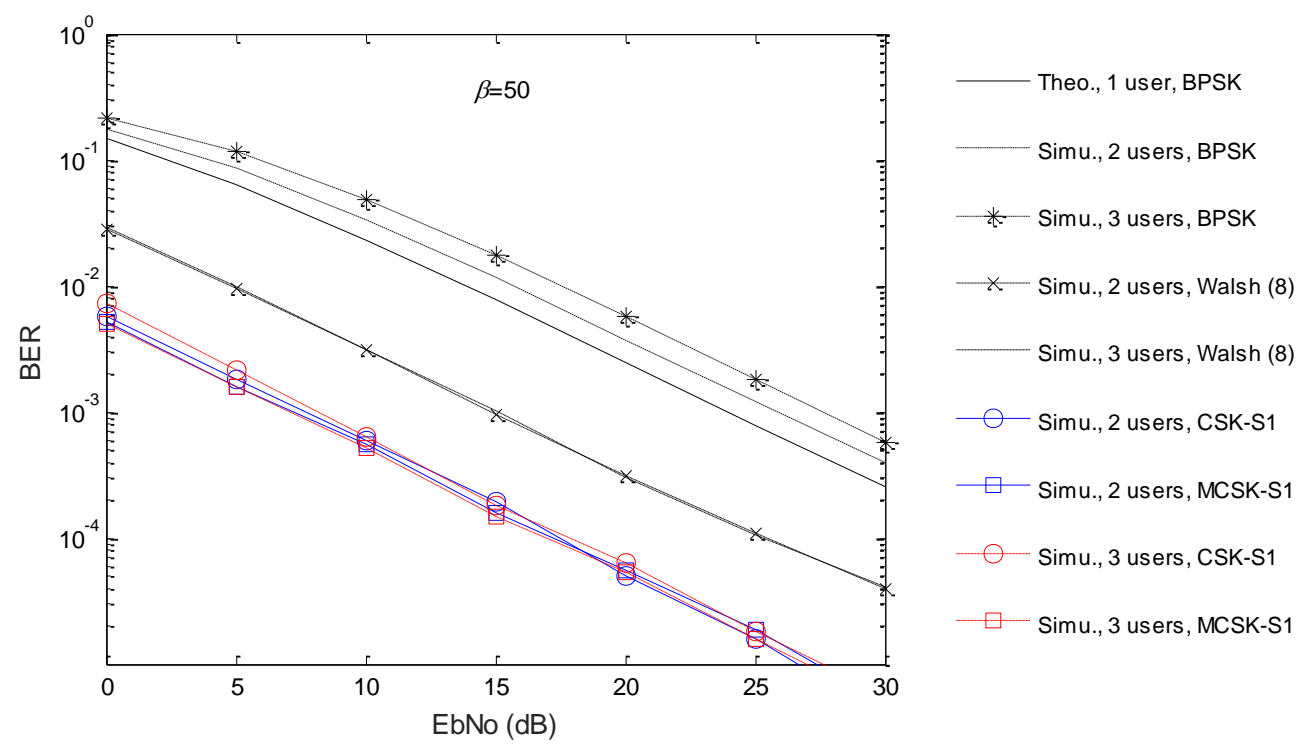

Fig. 6. Average BER performance of 2 and 3 users employing CSK-S1 and MCSK-S1 with $\beta=50$ in CB-NOMA over Rayleigh fading channel compared with the reference systems.
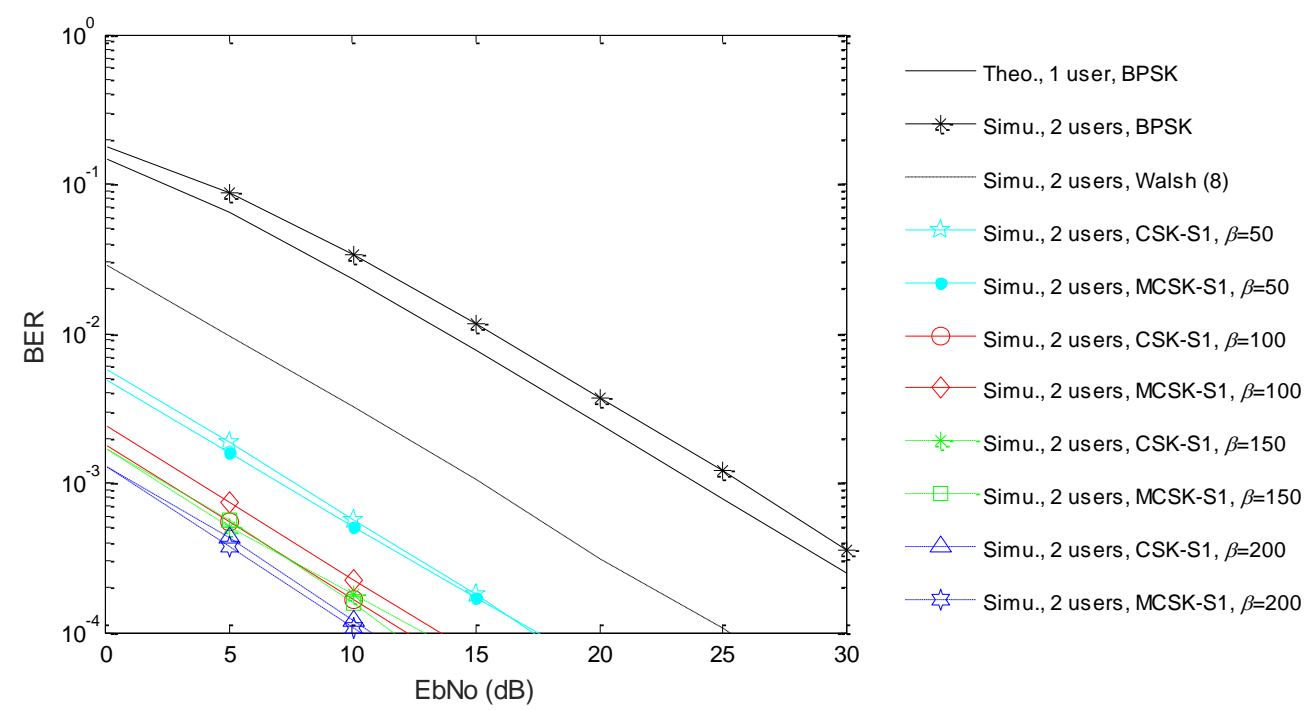

Fig. 7. Average BER performance of 2 users employing CSK-S1 and MCSK-S1 with different values of $\beta$ in CB-NOMA over Rayleigh fading channel compared with the reference systems. 
In Figure 6, the average BER results of CB-NOMA are presented as a function of SNR for 2 and 3 users considering CSK-S1 and MCSK-S1 with $\beta=50$. The close results for considered configurations and both cases of connected users ( 2 and 3 users) demonstrate the robust BER performance of CB-NOMA with the increased number of users similar to the conventional CDMA. This is an interesting issue since the number of allowed nonorthogonal users in this scenario is only limited by the target BER rather than the available number of orthogonal codes in the reference CDMA scheme.

The impact of using different $\beta$ values on the BER performance of 2 users CB-NOMA with CSK-S1 and MCSK-S1 modulation methods is presented in Figure 7. As can be seen, the employment of larger $\beta$ (spreading code length) is reflected by an improved BER as well as the required information security and other important characteristics provided by the chaotic signals. For instance, SNR gain of $7 \mathrm{~dB}$ at target BER of $10^{-4}$ is achieved when the code length is increased from $\beta=50$ to $\beta=200$.

Table 2. Summary of the approximate SNR gains of investigated scenarios (S1-S4) of CB-NOMA with $\beta=50$ and 100, $K=2$ and 3 users, and for both CSK and MCSK modulation methods in Figures 4 and 5 compared with the reference Walsh-Hadamard CDMA at target BER of $10^{-4}$.

\begin{tabular}{c|c|c|c|c}
\hline \multirow{2}{*}{$\begin{array}{c}\text { CB-NOMA } \\
\text { Scenario }\end{array}$} & \multicolumn{2}{|c|}{ SNR $(\mathrm{dB})$ for the case of $\beta=50$} & \multicolumn{2}{c}{ SNR $(\mathrm{dB})$ for the case of $\beta=100$} \\
\cline { 2 - 5 } & $K=2$ users & $K=3$ users & $K=2$ users & $K=3$ users \\
\hline CSK-S1 & 8 & 7 & 13 & 13.5 \\
\hline CSK-S2 & 10 & 10 & 13 & 14 \\
\hline CSK-S3 & 10 & 10 & 8.5 & 13.5 \\
\hline CSK-S4 & 6 & 7 & 8.4 & 10 \\
\hline MCSK-S1 & 9 & 7 & 11 & 10 \\
\hline MCSK-S2 & 8.5 & 8 & 11 & 10 \\
\hline MCSK-S3 & 8.5 & 8 & 11 & 8.5 \\
\hline MCSK-S4 & 6 & 6 & 8.4 &
\end{tabular}

\section{Conclusion}

In this paper, an efficient design of uplink CB-NOMA communication scheme has been presented over realistic fading channel by exploiting the advantages of physical layer security incoherent CBSCs and high user connectivity of code-domain NOMA. Different code formation scenarios, S1-S4, are considered for CSK and MCSK signalling and evaluated using joint ML detection. The achieved simulation results of BER validated the effectiveness of CB-NOMA compared with the benchmark references. Moreover, advantageous tradeoffs can be realized between the required error performance, security level, connectivity, and complexity. For instance, at BER of $10^{-4}$, the error rate of 3 users system with low complexity CSK-S2 and acceptable security outperforms that of CSK-S4 with high complexity and security by about $3 \mathrm{~dB}$ for $\beta=50$ and $5.4 \mathrm{~dB}$ for $\beta=100$. The interesting outcomes of CB-NOMA may lead to extend the paradigm for upcoming secure wireless communications. In future work, both of the code-domain and power-domain NOMA strategies will be integrated with CBSC systems to capture most of the promised gains of these schemes such as the high connectivity with diverse quality of services. 


\section{References}

[1] Dai L., Wang B., Yuan Y., Han S., I C-L, Wang Z.: Non-orthogonal multiple access for 5G: solutions, challenges, opportunities, and future research trends. IEEE Commun. Mag., Vol. 53, No. 9, pp. 74-81 (2015)

[2] Dai L., Wang B., Ding Z., Wang Z., Chen S., Hanzo L.: A survey of non-orthogonal multiple access for 5G. IEEE Commun. Surveys and Tutorials. Vol. 20, No. 3, pp. 2294-2323 (2018)

[3] Al-Hussaibi W., Ali F.: Efficient user clustering, receive antenna selection, and power allocation algorithms for massive MIMO-NOMA systems. IEEE Access. Vol. 7, No. 6, pp. 31865-31882 (2019) [4] Sari H., Maatouk A., Caliskan E., Assaad M., Koca M., Gui G.: On the foundation of NOMA and its Application to 5G cellular networks. Proc. IEEE Wireless Communications and Networking Conference (WCNC). pp. 1-6, Barcelona, Spain (2018)

[5] Al-Hussaibi W.: Optimal cluster formation and power control for high connectivity wireless MIMONOMA applications. Electronics Letters. Vol. 55, No.20, pp. 1110-1112 (2019)

[6] Sari H., Vanhaverbeke F., Moeneclaey M.: Extending the capacity of multiple access channels. IEEE Commun. Mag. Vol. 38, pp. 74-82 (2000)

[7] Maatouk A., Caliskan E., Koca M., Assaad M., Gui G., Sari H.: Frequency-domain NOMA with two sets of orthogonal signal waveforms. IEEE Commun. Letters. Vol. 22, No. 5, pp. 906-909 (2018) [8] Horiike N., Okamoto E., Yamamoto T.: A downlink non-orthogonal multiple access scheme having physical layer security. EURASIP Journal on Wireless Communications and Networking. Vol. 2018, No. 205, pp. 1-13 (2018)

[9] Okamoto E., Horiike N., Yamamoto T.: Sparse chaos code multiple access scheme achieving larger capacity and physical layer security. Proc.20th International Symposium on Wireless Personal Multimedia Communications (WPMC). pp. 604-610, Bali, Indonesia (2017)

[10] Masuda Y., Okamoto E., Ito K., Yamamoto T.: An uplink non-orthogonal multiple access scheme having physical layer security based on chaos modulation. Proc. International Conference on Information Networking (ICOIN). pp. 136-140, Kuala Lumpur, Malaysia (2019)

[11] Okamoto E.: A chaos MIMO transmission scheme for secure communications on physical layer. Proc. IEEE 73rd Vehicular Technology Conference (VTC Spring). pp. 1-5, Yokohama, Japan (2011)

[12] Ren H., Baptista M., Grebogi C.: Wireless communication with chaos. Physical Review Letter. Vol. 110, No. 18, pp. 1-5 (2013)

[13] Al-Hussaibi W., Alsmael J., Türkmen M.: On the chaos synchronization in CBSC systems over realistic wireless channels. Proc. 16th SSD'19-CSP. pp. 654-659, Istanbul, Turkey (2019)

[14] Eisencraft M., Fanganiello R., Grzybowski J., Soriano D., Attux R., Batista A., Macau E., Monteiro L., Romano J., Suyama R., Yoneyama T.: Chaos-based communication systems in non-ideal channels. Communications in Nonlinear Science and Numerical Simulation. Vol. 17, No. 12, pp. 4707-4718 (2012)

[15] Al-Hussaibi W.: Effect of filtering on the synchronization and performance of chaos-based secure communication over Rayleigh fading channel. Communications in Nonlinear Science and Numerical Simulation. Vol. 26, No. 1-3, pp. 87-97 (2015)

[16] Kaddoum G.: Wireless chaos-based communication systems: a comprehensive survey. IEEE Access. Vol. 4, pp. 2621-2648 (2016)

[17] Tam W., Lau F., Tse C., Yip M.: An approach to calculating the bit-error rate of a coherent chaosshift-keying digital communication system under a noisy multiuser environment. IEEE Transactions on Circuits and Systems I: Fundamental Theory and Applications. Vol.49, No. 2, pp. 210-223 (2002)

[18] Tam W., Lau F., Tse C.: A multiple access scheme for chaos-based digital communication systems utilizing transmitted reference. IEEE Transactions on Circuits and Systems I: Regular Papers. Vol. 51, No,9, pp.1868-1878 (2004)

[19] Yang T., Chua L.: Chaotic digital code-division multiple access (CDMA) communication systems. International Journal of Bifurcation and Chaos. Vol. 7, No. 12, pp. 2789-2805 (1997)

[20] Harb A., Al-Hussaibi W., Khadra L.: Effects of filtering chaotic signals of power electronic circuit. International Journal of Modeling and Simulation. Vol. 23, No. 2, pp. 129-134 (2003) 